\title{
Surface termination dependent work function and electronic properties of $\mathrm{Ti}_{3} \mathrm{C}_{2} \mathrm{~T}_{\mathrm{x}}$ MXene
}

\author{
Thorsten Schultz ${ }^{1,2^{*}}$, Nathan C. Frey ${ }^{3 *}$, Kanit Hantanasirisakul ${ }^{4,5^{*}}$, Soohyung \\ Park $^{1,6}$, Steven J. May ${ }^{5}$, Vivek B. Shenoy ${ }^{3}$, Yury Gogotsi ${ }^{4,5}$, Norbert Koch ${ }^{1,2 \#}$ \\ ${ }^{1}$ Institut für Physik \& IRIS Adlershof, Humboldt-Universität zu Berlin, Berlin, Germany \\ ${ }^{2}$ Helmholtz-Zentrum Berlin für Materialien und Energie GmbH, Berlin, Germany \\ ${ }^{3}$ Department of Materials Science and Engineering, University of Pennsylvania, Philadelphia, \\ USA \\ ${ }^{4}$ A.J. Drexel Nanomaterials Institute, Drexel University, Philadelphia, USA \\ ${ }^{5}$ Department of Materials Science and Engineering, Drexel University, Philadelphia, USA \\ ${ }^{6}$ Advanced Analysis Center, Korea Institute of Science \& Technology, Seoul, Korea \\ * These authors contributed equally to this work. \\ \# Corresponding author email: nkoch@physik.hu-berlin.de
}

\begin{abstract}
MXenes, an emerging family of 2D transition metal carbides and nitrides, have shown promise in various applications, such as energy storage, electromagnetic interference shielding, conductive thin films, photonics, and photothermal therapy. Their metallic nature, wide range of optical absorption, and tunable surface chemistry are the key to their success in those applications. The physical properties of MXenes are known to be strongly dependent on their surface terminations. In this study we investigated the electronic properties of $\mathrm{Ti}_{3} \mathrm{C}_{2} \mathrm{~T}_{\mathrm{x}}$ for different surface terminations, as achieved by different annealing temperatures, with the help of photoelectron spectroscopy, inverse photoelectron spectroscopy, and density functional theory calculations. We find that fluorine occupies solely the face-centered cubic adsorption site, whereas oxygen initially occupies at least two different adsorption sites, followed by a rearrangement after fluorine desorption at high annealing temperatures. The measured electronic structure of $\mathrm{Ti}_{3} \mathrm{C}_{2} \mathrm{~T}_{\mathrm{x}}$ showed strong dispersion of more than $1 \mathrm{eV}$, which we conclude to stem from Ti-O bonds by comparing it to calculated band structures. We further measured the work function of $\mathrm{Ti}_{3} \mathrm{C}_{2} \mathrm{~T}_{\mathrm{x}}$ as a function of annealing temperature and found that is in the range of 3.9-4.8 eV, depending on the surface composition. Comparing the experimental work function to detailed density functional theory calculations shows that the measured value is not simply an average of the work function values of uniformly
\end{abstract}


terminated $\mathrm{Ti}_{3} \mathrm{C}_{2}$ surfaces, but that the interplay between the different surface moieties and their local dipoles plays a crucial role.

\section{Introduction}

2D materials received tremendous attention over the past decade due to their interesting properties different from their bulk counterparts, which render them promising in a variety of applications from energy storage to photonics. ${ }^{1}$ Typical 2D materials include graphene, transition metal dichalcogenides (TMDCs), transition metal oxides, halides and hydroxides, and hexagonal boron nitride. ${ }^{2}$ Since 2011, a large family of 2D transition metal carbides and nitrides, so-called MXenes, was added to the $2 \mathrm{D}$ world. ${ }^{3}$ MXenes have a general formula of $\mathrm{M}_{\mathrm{n}+1} \mathrm{X}_{\mathrm{n}} \mathrm{T}_{\mathrm{x}}$, where $\mathrm{M}$ is an early transition metal such as $\mathrm{Ti}, \mathrm{V}, \mathrm{Mo}, \mathrm{Nb}$, whereas $\mathrm{X}$ is $\mathrm{C}$ and/or $\mathrm{N}$, and $\mathrm{T}_{\mathrm{x}}$ represents surface terminations such as $\mathrm{OH}, \mathrm{O}$ and $\mathrm{F}$. MXenes are usually produced from selective etching of the $\mathrm{A}$ layer from MAX phases, ${ }^{4}$ where A is a group III to VI element, typically $\mathrm{Al}$ or $\mathrm{Si}^{5}{ }^{5}$ Close to 30 MXenes have been produced to date, making them one of the largest and rapidly growing families of $2 \mathrm{D}$ materials. ${ }^{6}$ Due to their metallic conductivity and versatile chemistry, they have shown promise in many applications, such as energy storage, ${ }^{7}$ electromagnetic interference shielding, ${ }^{8}$ sensors, ${ }^{9,} 10$ and photothermal therapy. ${ }^{11}$ However, little is known about their fundamental electronic properties, in particular the correlation between surface termination and the MXene electronic structure. Only a handful of experiments have been reported in efforts to understand these properties, despite several theoretical calculations. ${ }^{12,13}$ Density functional theory (DFT) studies predicted a strong influence of the surface termination type on MXenes' work function, ${ }^{14}$ metal-to-insulator transition, ${ }^{15}$ and electronic band occupation, ${ }^{16}$ all of which await experimental confirmation. Reports on photoelectron spectroscopy investigations of the electronic structure of $\mathrm{Ti}_{3} \mathrm{C}_{2}$ are scarce, ${ }^{17}$ and only limited experimental data on the work function of this material are available. ${ }^{18}$ In the present study, we provide direct evidence of the effects of surface termination on the work function and electronic band structure of $\mathrm{Ti}_{3} \mathrm{C}_{2} \mathrm{~T}_{\mathrm{x}}$, one of the most widely studied MXenes, using a combination of ultraviolet photoemission spectroscopy (UPS), X-ray photoelectron spectroscopy (XPS) and inverse photoemission spectroscopy (IPES). By comparing our experimental results to DFT calculations we determine the adsorption sites of the different surface terminations. This work not only provides direct experimental evidence of the effects of surface terminations on MXenes' electronic properties, but also lays a ground for using spectroscopic techniques to study electronic properties of other MXenes. Understanding and, ultimately, being able to control their electronic properties are the key to the success in using MXenes in different applications.

\section{Methods}

\section{Sample Preparation}

$\mathrm{Ti}_{3} \mathrm{AlC}_{2}$ (Carbon-Ukraine) was etched in a mixture of $\mathrm{LiF}$ and $\mathrm{HCl}$ following a protocol reported elsewhere. ${ }^{19}$ Briefly, $0.5 \mathrm{~g}$ of the MAX powder was added slowly to a premixed solution of $0.8 \mathrm{~g}$ of $\mathrm{LiF}$ and $10 \mathrm{~mL}$ of $9 \mathrm{M} \mathrm{HCl}$. The mixture was stirred at room temperature $\left(25^{\circ} \mathrm{C}\right)$ for $24 \mathrm{~h}$. Then it was washed repeatedly 4-5 times by adding $150 \mathrm{~mL}$ of DI water to the mixture, centrifuge for 5 min, and decant the supernatant. When the $\mathrm{pH}$ of the solution was close to neutral, dark supernatant was observed indicating delamination of MXene flakes $\left(\mathrm{d}-\mathrm{Ti}_{3} \mathrm{C}_{2} \mathrm{~T}_{\mathrm{x}}\right)$. The first dark supernatant was 
decanted, and $25 \mathrm{~mL}$ of DI water was added to the sediment. The mixture was shaken vigorously for $15 \mathrm{~min}$ to improve delamination yield. Finally, the mixture was centrifuged at $3500 \mathrm{rpm}$ for 30 min to collect $\mathrm{d}-\mathrm{Ti}_{3} \mathrm{C}_{2} \mathrm{~T}_{\mathrm{x}}$ in the supernatant. MXene thin films were prepared by spin-casting using a spin-coater (Laurell Technologies, Model WS-650 Hz, USA). Approximately $10 \mathrm{mg} / \mathrm{mL}$ of d$\mathrm{Ti}_{3} \mathrm{C}_{2} \mathrm{~T}_{\mathrm{x}}$ was applied to a plasma-treated quartz substrate and spun at $2000 \mathrm{rpm}$ for $30 \mathrm{~s}$. The films were subsequently dried at $5000 \mathrm{rpm}$ for $15 \mathrm{~s}$, and the process was repeated two times to obtain films with appropriate thickness.

\section{Photoelectron Spectroscopy and Inverse Photoelectron Spectroscopy}

Photoelectron spectroscopy measurements were performed at Humboldt University of Berlin, using two combined preparation and analysis chambers with base pressures of less than $10^{-8}$ mbar. XPS spectra were recorded using the radiation of a Mg source $(1253.6 \mathrm{eV})$ and UPS spectra were recorded using the radiation of a He discharge lamp $(21.21 \mathrm{eV})$. The kinetic energy of the emitted electrons was detected by a Specs Phoibos 100 hemispherical analyzer with a resolution of $\approx 150 \mathrm{meV}$ and an angular acceptance angle of $\pm 3^{\circ}$ in the UPS measurements. During the measurements of the secondary electron cut-off, a bias of $-10 \mathrm{~V}$ was applied between the sample and the analyzer. The XPS spectra were fitted with CasaXPS, using a Shirley-background, asymmetric line profiles for peaks stemming from the MXene (due to the interaction of the photoelectrons with delocalized electrons) and Voigt line shapes for peaks stemming from contaminations. Inverse photoelectron spectroscopy (IPES) measurements were performed in the isochromat mode, using incident electron energies of 5-15 eV and a $\mathrm{NaCl}$-coated photocathode for detecting the emitted photons. The resolution of the setup was determined to be $1.3 \mathrm{eV}$. The samples were resistively heated in ultrahigh vacuum inside the XPS chamber during the course of the experiment and the spectra were measured at room temperature.

\section{Density Functional Theory}

The Vienna Ab-Initio Simulation Package (VASP) ${ }^{20}$ was used for all DFT calculations. Structural relaxations were performed with the Perdew-Burke-Ernzerhof (PBE) ${ }^{21}$ exchange-correlation functional and projector augmented wave (PAW) pseudopotentials, ${ }^{22}$ with a $520 \mathrm{eV}$ plane-wave basis cutoff, a 10x10x1 $\Gamma$ centered $k$-point mesh, and forces on each atom converged to within $10^{-2} \mathrm{eV} / \AA$. We assumed non-spin polarized ground states, following previous predictions of nonmagnetic functionalized $\mathrm{Ti}_{3} \mathrm{C}_{2} \mathrm{~T}_{\mathrm{x}}{ }^{23,24} \mathrm{To}$ compute accurate band structures, the Heyd-ScuseriaErnzerhof (HSE06) hybrid functional was used. ${ }^{25,26}$ Due to the significant computational cost of hybrid functional calculations a $7 \times 7 \times 1 \Gamma$ centered $k$-point mesh was used for the HSE06 starting point, and work functions and other electronic properties were calculated for supercells using the SCAN meta-GGA functional after appropriately reducing the $k$-point mesh to preserve the $k$-point density. ${ }^{27,28}$ The core level binding energy shifts are estimated from DFT in the initial state approximation. Because the calculated absolute energies are not relevant for comparison with experiment, only relative energy shifts are reported. The density of states (DOS) obtained from DFT was broadened with a Gaussian function with a full width at half maximum (FWHM) of $0.4 \mathrm{eV}$ and $1.0 \mathrm{eV}$ for comparison with UPS and IPES data, respectively.

Temperature Programmed Desorption 
Thermogravimetric analysis and mass spectroscopy were performed using a Discovery SDT 650 model connected to a Discovery mass spectrometer (TA Instruments, DE). The MXene freestanding 'paper' was made from the same MXene solution used in thin film fabrication via a vacuum-assisted filtration method. The paper with a mass of $\approx 10 \mathrm{mg}$ was dried in a vacuum desiccator at least $24 \mathrm{~h}$ prior to the measurement. Then it was packed in a $90 \mu \mathrm{L}$ alumina pan and heated to $1500{ }^{\circ} \mathrm{C}$ at a constant heating rate of $10{ }^{\circ} \mathrm{C} / \mathrm{min}$ in $\mathrm{He}$ atmosphere $(100 \mathrm{~mL} / \mathrm{min})$. The furnace was purged with $100 \mathrm{~mL} / \mathrm{min}$ flow of $\mathrm{He}$ gas for one hour before the analysis to remove air residual.

\section{Dynamic Light Scattering (DLS)}

Delaminated $\mathrm{Ti}_{3} \mathrm{C}_{2} \mathrm{~T}_{\mathrm{x}}$ solution with a concentration around $0.01 \mathrm{mg} / \mathrm{mL}$ was analyzed using a Zetasizer Nano ZS (Malvern, Panalytical) in a polystyrene cuvette. The average particle size was taken over a total of three measurements from each sample.

\section{Atomic Force Microscopy (AFM)}

AFM images were recorded with a Bruker AFM Multimode 8 in tapping mode using a driving frequency of $300 \mathrm{kHz}$, driving amplitude of $80 \mathrm{mV}$, and scanning frequency between 0.6 and 0.9 $\mathrm{Hz}$.

\section{Results and Discussion}

To identify the adsorption site of the different surface terminations and the resulting impact on the electronic structure, XPS, UPS, and IPES measurements for $\mathrm{Ti}_{3} \mathrm{C}_{2} \mathrm{~T}_{\mathrm{x}}$ annealed at different temperatures were compared together with DFT calculations. Figure 1 shows the different adsorption sites for surface terminations considered in this work. Figure 1a) illustrates a top view

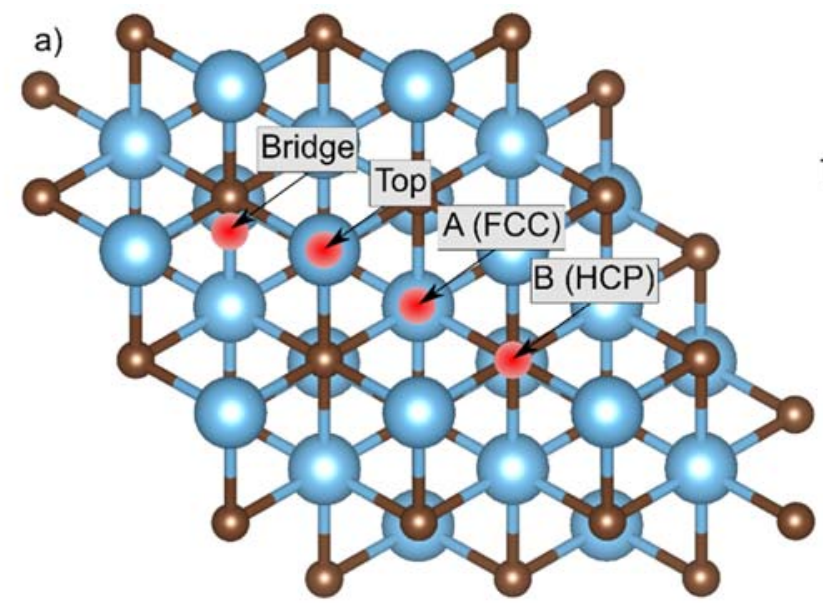

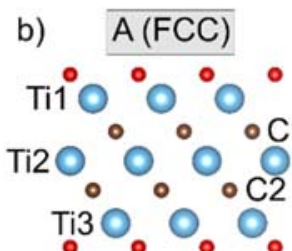

c)

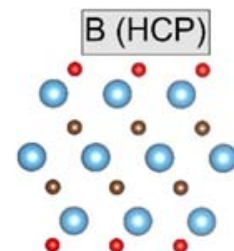

d)

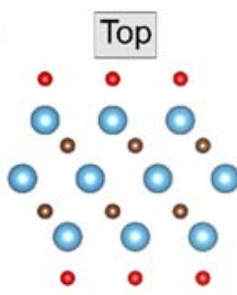

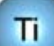

e)

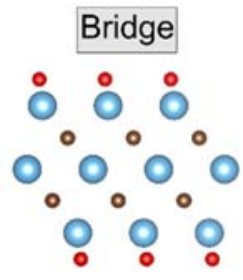

Figure 1: Schematic illustration of the different adsorption sites of $\mathrm{Ti}_{3} \mathrm{C}_{2} \mathrm{~T}_{\mathrm{x}}$ considered for the DFT calculations in this work. a) Top view and side views of b) A (FCC) adsorption site (on top of Ti atom in the third atomic layer), c) B (HCP) adsorption site (on top of $\mathrm{C}$ atom in the second atomic layer), d) top adsorption site (on top of a $\mathrm{Ti}$ atom in the first atomic layer) and e) bridge adsorption site (between topmost $\mathrm{Ti}$ and $\mathrm{C}$ atoms). 
of the $\mathrm{Ti}_{3} \mathrm{C}_{2}$ lattice, with the different adsorption sites marked in red, while Figures $1 \mathrm{~b}$ )-e) show the side view of the different adsorption sites. The A (FCC) site shown in b) marks the surface termination adsorbed directly above the Ti atoms in the third atomic layer (Ti2). The B (HCP) site shown in c) marks the surface termination adsorbed directly above the carbon atoms in the second atomic layer (C1). The top site shown in d) marks the surface terminations adsorbed directly above the Ti atoms in the first atomic layer (Til) and the bridge site shown in e) marks the surface terminations adsorbed between the topmost $\mathrm{Ti}$ and $\mathrm{C}$ atoms. These labels will be used throughout this report.

The temperature dependent XPS measurements reveal clear changes in the $\mathrm{O} 1 \mathrm{~s}$ and $\mathrm{C} 1 \mathrm{~s}$ core level spectra between room temperature and $470{ }^{\circ} \mathrm{C}$, accompanied by a sharpening of the valence band as observed in UPS valence spectra. Above $500{ }^{\circ} \mathrm{C}$ desorption of fluorine is observed, which goes in hand with an intensity redistribution in the O1s core level. An overview of the XPS core level and UPS valence band evolution for different annealing temperatures is shown in the supplementary information (SI) Figure S1. Detailed XPS spectra of the C1s, Ti2p, O1s and F1s core levels for three representative annealing temperatures (as loaded, $470{ }^{\circ} \mathrm{C}$, and $750{ }^{\circ} \mathrm{C}$ ) are shown in Figure 2. The C1s core level [Figure 2 a)] of the as-loaded $\mathrm{Ti}_{3} \mathrm{C}_{2} \mathrm{~T}_{\mathrm{x}}$ consists of four components. One from the Ti-C bonds of the MXene (purple) at $281.9 \mathrm{eV}$, one from $\mathrm{C}-\mathrm{C}$ contaminations (green) at around $284.9 \mathrm{eV}$, and two stemming from $\mathrm{C}-\mathrm{O}$ (blue) and $\mathrm{O}-\mathrm{C}=\mathrm{O}$ (orange) contaminations at $286.2 \mathrm{eV}$ and $288.9 \mathrm{eV}$, respectively. With increasing annealing temperature, the contaminations desorb. At $470{ }^{\circ} \mathrm{C}$ the $\mathrm{C}-\mathrm{O}$ and $\mathrm{O}-\mathrm{C}=\mathrm{O}$ contaminations are completely removed, only $\mathrm{C}-\mathrm{C}$ contaminations remain up to $750{ }^{\circ} \mathrm{C}$, in agreement with previous reports. ${ }^{29,30}$ The Ti2p peaks shown in Figure $2 b$ ) consist of three doublets (spin-orbit splitting). The doublet at $455.2 \mathrm{eV}$ and $461.1 \mathrm{eV}$ (purple) stems from Ti-C bonds of the MXene, the doublet at $456.2 \mathrm{eV}$ and $461.6 \mathrm{eV}$ stems from Ti-O (blue) and the doublet at $457.3 \mathrm{eV}$ and $462.7 \mathrm{eV}$ stems from Ti-F (red), respectively. The absence of a peak at around $459 \mathrm{eV}$ indicates that no $\mathrm{TiO}_{2}$ has formed during the sample preparation or the annealing process. ${ }^{31,32}$ No significant change in the Ti2 $p$ spectra is observed for all annealing temperatures. Figure $2 c$ ) shows the O1s spectra, where we observe that the spectra for the as-loaded sample consists of at least five peaks, of which the peaks at $529.8 \mathrm{eV}$ (purple) and $531.6 \mathrm{eV}$ (red) are assigned to oxygen bound to titanium at different lattice sites. Calculation of the relative binding energy shifts by DFT (Table S1 in the SI) suggests that the peak at $529.8 \mathrm{eV}$ stems from oxygen on a bridge site and the peak at $531.6 \mathrm{eV}$ either to oxygen at the B site or to oxygen on the A site in the presence of fluorine, as suggested by Persson and coworkers. ${ }^{29}$ However, considering the relative formation energies for the different adsorption sites (Table S2 in the SI), only oxygen on the top adsorption site can be confidently excluded. The blue peak at $532.5 \mathrm{eV}$ is assigned to $\mathrm{C}-\mathrm{O}$ contaminations and the orange peak at $533.4 \mathrm{eV}$ to $\mathrm{C}=\mathrm{O}$ contaminations, in agreement with the $\mathrm{C} 1 \mathrm{~s}$ core level signal. The light blue peak at $533.8 \mathrm{eV}$ could be due to adsorbed water or $\mathrm{OH}$ surface termination groups. ${ }^{33}$ Similar to the $\mathrm{C} 1 \mathrm{~s}$ core level, the $\mathrm{C}$ $\mathrm{O}$ and $\mathrm{C}=\mathrm{O}$ contaminations as well as the $\mathrm{OH}$ terminations are completely removed after annealing at $470{ }^{\circ} \mathrm{C}$. This is in good agreement with observations from temperature programmed desorption measurements (Figure S2 in the SI). After annealing at $750{ }^{\circ} \mathrm{C}$, the $\mathrm{O} 1 \mathrm{~s}$ peak becomes broader and can only be fitted adequately by adding a third peak at $530.7 \mathrm{eV}$ (dark yellow). The F1s core level shown in Figure $2 \mathrm{~d}$ ) consists of one peak assigned to Ti-F surface termination (purple) at $685.2 \mathrm{eV}$ and a small peak at $687.1 \mathrm{eV}$, which can be assigned to F-containing contaminations from the 
etching of the MAX phase. ${ }^{34,35}$ The fluorine starts to desorb above $500{ }^{\circ} \mathrm{C}$ and is reduced by about $60-80$ at. $\%$ at $750{ }^{\circ} \mathrm{C}$, simultaneous to the emergence of the new peak in the $\mathrm{O} 1 \mathrm{~s}$ spectrum. Persson et al. assigned this peak to oxygen on the A-site in the absence of surrounding fluorine, ${ }^{29}$ in agreement with our calculated relative binding energy shifts. From the survey spectra (Figure S3 in the SI) it can be observed that a very small amount of $\mathrm{Cl}(<2 \%)$, from the $\mathrm{LiF}+\mathrm{HCl}$ etchant, is present at all temperatures.

We now discuss the electronic structure of $\mathrm{Ti}_{3} \mathrm{C}_{2} \mathrm{~T}_{\mathrm{x}}$ as inferred from UPS. The valence band spectrum of as-loaded $\mathrm{Ti}_{3} \mathrm{C}_{2} \mathrm{~T}_{\mathrm{x}}$ (Figure $\mathrm{S} 1$ ) is dominated by carbon contaminations and shows little features. After desorption of the contaminations, the valence band becomes sharper and shows a distinct Fermi-edge, which is a clear indication for the metallic character of the $\mathrm{Ti}_{3} \mathrm{C}_{2} \mathrm{~T}_{\mathrm{x}}$. Features around $2 \mathrm{eV}, 3.5 \mathrm{eV}, 6 \mathrm{eV}$ and $9 \mathrm{eV}$ binding energy are also observed. The valence band spectra, after background subtraction and normalization (see Figure S4 in the SI for details), are shown in Figure 3a) for two different surface terminations $\left(\mathrm{Ti}_{3} \mathrm{C}_{2} \mathrm{~F}_{0.8} \mathrm{O}_{0.8}\right.$, solid lines, and $\mathrm{Ti}_{3} \mathrm{C}_{2} \mathrm{~F}_{0.2} \mathrm{O}_{0.8}$, dashed lines). After annealing at $750^{\circ} \mathrm{C}$, a strong decrease of the fluorine $\mathrm{F} 1 \mathrm{~s}$ signal was observed in XPS [see Figure 2d)], which goes along with a decrease of the valence band peak at around $9 \mathrm{eV}$ as well as the peak close to the Fermi-level (set to $0 \mathrm{eV}$ binding energy). Therefore, we assign these two features to DOS associated with fluorine surface termination. A comparison to the calculated total DOS of $\mathrm{Ti}_{3} \mathrm{C}_{2} \mathrm{~F}_{2}$ shown in Figure $3 \mathrm{~b}$ ), as well as the single XPS peak in the F1s core level

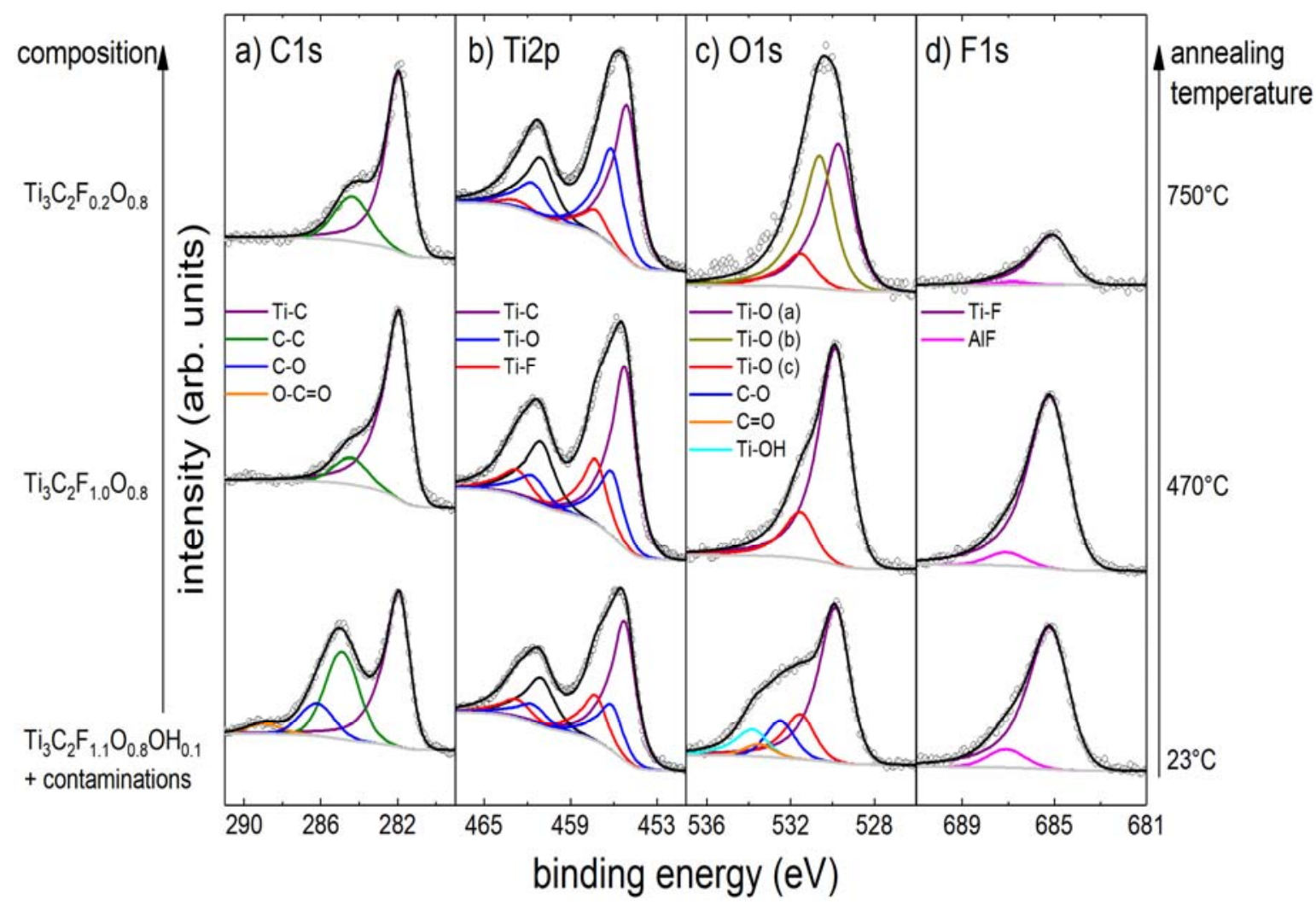

Figure 2: XPS core level spectra a) $\mathrm{C} 1 \mathrm{~s}$, b) Ti2p, c) O1s and d) $\mathrm{F}_{1} \mathrm{~s}$ of $\mathrm{Ti}_{3} \mathrm{C}_{2} \mathrm{~T}_{\mathrm{x}}$, obtained for asloaded sample (lower row), after annealing at $470{ }^{\circ} \mathrm{C}$ (middle row) and after annealing at $750{ }^{\circ} \mathrm{C}$. (upper row). Hollow circles represent measured data points and solid lines represent curve fits. The estimated surface terminations are shown on the left. 
[Figure 2d)], clearly identifies the fluorine to be solely adsorbed on the A-site. This is in agreement with findings by Persson and coworkers. ${ }^{29}$ However, a clear assignment of the oxygen adsorption site cannot be made from a comparison between the measured valence band and the calculated density of states of $\mathrm{Ti}_{3} \mathrm{C}_{2} \mathrm{O}_{2}$ shown in Figure 3c), as the total DOS for the A-, B- and bridge-site are very similar. Only the top adsorption site can be ruled out, as the intensity around $6 \mathrm{eV}$, which stems from oxygen termination, is not present in the total DOS for the top-site. This agrees with the high formation energy of this adsorption site, as mentioned before. It should be mentioned at this point that a direct comparison between measured UPS spectra and calculated DOS from DFT must be interpreted with care, as the Kohn-Sham approximation applied in DFT neglects many body effects.

Figure 3a) shows additional UPS spectra for different photoelectron momentum values parallel to the sample surface $(\mathrm{k} \|$, corresponding to different take-off angles). There is an obvious angular dependence in the features around $2 \mathrm{eV}$, which is an indication for band dispersion. Normally the observation of band dispersion is only expected for single crystalline samples. However, there have been examples of non-single crystalline graphite and transition metal dichalcogenides (TMDCs), which showed dispersive bands in angle-resolved photoemission measurements (ARPES) ${ }^{36,37}$ The high symmetry of the crystal lattice and the particular azimuthal photoemission-intensity dependence enable a measurement of the dispersion, which is dominated by the high symmetry directions $(\Gamma-\mathrm{M}$ and $\Gamma-\mathrm{K}$, for a more detailed explanation see Figure S5). A curvature plot, as obtained from the measured ARPES data by following a procedure described by Zhang et al., ${ }^{38}$ for a $\mathrm{Ti}_{3} \mathrm{C}_{2} \mathrm{~F}_{0.8} \mathrm{O}_{0.8}$ sample is shown in Figure 4a). Non-dispersive bands are visible around $5.5 \mathrm{eV}$ and $9 \mathrm{eV}$, corresponding to oxygen and fluorine surface terminations, respectively, as discussed earlier. Notably, rather dispersive bands can be seen from about $1.5 \mathrm{eV}$ binding energy to about $3 \mathrm{eV}$ binding energy, when moving from $\mathrm{k}_{\|}=0 \AA^{-1}$ to $\mathrm{k}_{\|}=1.2 \AA^{-1}$. Selected bands from the calculated band structure of $\mathrm{Ti}_{3} \mathrm{C}_{2} \mathrm{~F}_{2}$ with fluorine adsorbed at the A-site as well as $\mathrm{Ti}_{3} \mathrm{C}_{2} \mathrm{O}_{2}$ with oxygen adsorbed at the bridge-site are included in Figure 4a) for comparison and the complete band calculations are presented in Figure $4 \mathrm{~b}$ ). The calculated bands for the $\mathrm{Ti}_{3} \mathrm{C}_{2} \mathrm{~F}_{2}$ termination are in good agreement with the non-dispersive feature around $9 \mathrm{eV}$ and with the dispersive feature around the Fermi-level. The calculated bands for oxygen adsorbed at the bridge site agree fairly well with the

$$
\text { non-dispersive }
$$

feature

at

around

$5.5 \mathrm{eV}$ 


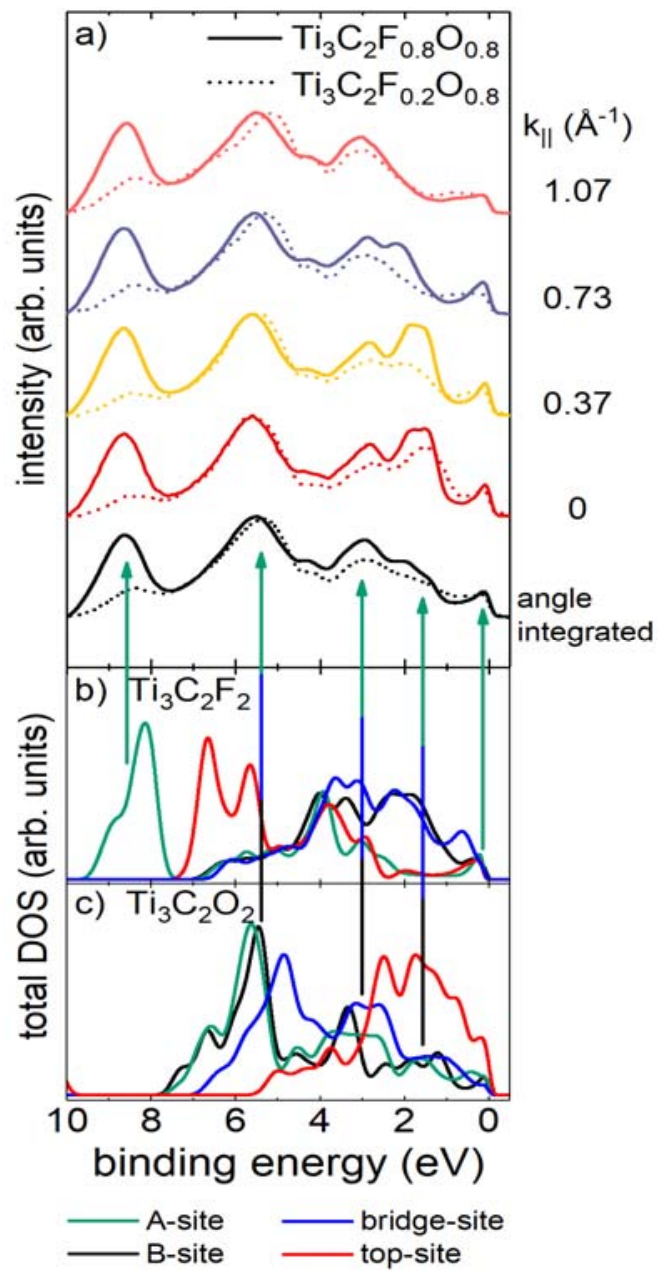

Figure 3: a) UPS valence band spectra of $\mathrm{Ti}_{3} \mathrm{C}_{2} \mathrm{~F}_{0.8} \mathrm{O}_{0.8}$ (solid lines) and $\mathrm{Ti}_{3} \mathrm{C}_{2} \mathrm{~F}_{0.2} \mathrm{O}_{0.8}$ (dashed lines) for different $\mathrm{k}_{\|}$and angle integrated. Dispersion can be observed between 1 and $3 \mathrm{eV}$ binding energy. b) and c) show the calculated total DOS for $\mathrm{Ti}_{3} \mathrm{C}_{2} \mathrm{~F}_{2}$ and $\mathrm{Ti}_{3} \mathrm{C}_{2} \mathrm{O}_{2}$ terminations at different adsorption sites. Arrows indicate the correlations between calculations and experimental data.

and with the dispersive features between 1 and $2.5 \mathrm{eV}$. All other calculated band structures derived from different adsorption sites of the surface terminations are shown in Figure S6, showing less agreement with the measured ARPES spectrum. However, the O1s peak consists of at least two peaks after removal of surface terminations and a third peak emerges after desorption of fluorine [dark yellow peak in Figure 2c)]. This new peak can be related to oxygen at the A-site in the absence of surrounding fluorine. This is also in agreement with findings by Persson and coworkers. ${ }^{29}$ Therefore, it is not surprising that the band structure calculated using only a single termination site cannot capture all the features observed in the ARPES measurements. 
We also performed IPES measurements on the $\mathrm{Ti}_{3} \mathrm{C}_{2} \mathrm{~F}_{0.8} \mathrm{O}_{0.8}$ and the $\mathrm{Ti}_{3} \mathrm{C}_{2} \mathrm{~F}_{0.2} \mathrm{O}_{0.8}$ samples to probe the unoccupied states, complementing the XPS and UPS measurements of the occupied states. The IPES spectra are shown in Figure S7 of the SI, together with the calculated total DOS. The IPES spectrum of the $\mathrm{Ti}_{3} \mathrm{C}_{2} \mathrm{~F}_{0.8} \mathrm{O}_{0.8}$ sample shows one peak at ca. $1.9 \mathrm{eV}$ binding energy, which decreases in intensity after fluorine desorption, as clearly observed when plotting the difference between the two spectra (grey). Comparison to the calculated DOS shown in Figure S7b) and c) suggests that this peak is in accordance with $\mathrm{F}$ adsorbed on the A-site, fully in line with the results obtained from XPS and UPS.

Knowing the surface composition and the most probable adsorption sites, we can now attend to the evolution of the $\mathrm{Ti}_{3} \mathrm{C}_{2} \mathrm{~T}_{\mathrm{x}}$ work function for different surface terminations, as the exact composition and adsorption site influence the calculated work function obtained from DFT. The work function of the $\mathrm{Ti}_{3} \mathrm{C}_{2} \mathrm{~T}_{\mathrm{x}}$, as determined from the secondary electron cut-offs [SECOs, shown in Figure 5a)], is summarized in Figure 5b) as a function of annealing temperatures (for exact composition at each temperature see SI Figure S8). The as-loaded $\mathrm{Ti}_{3} \mathrm{C}_{2} \mathrm{~T}_{\mathrm{x}}$ exhibits relatively low work function of ca.
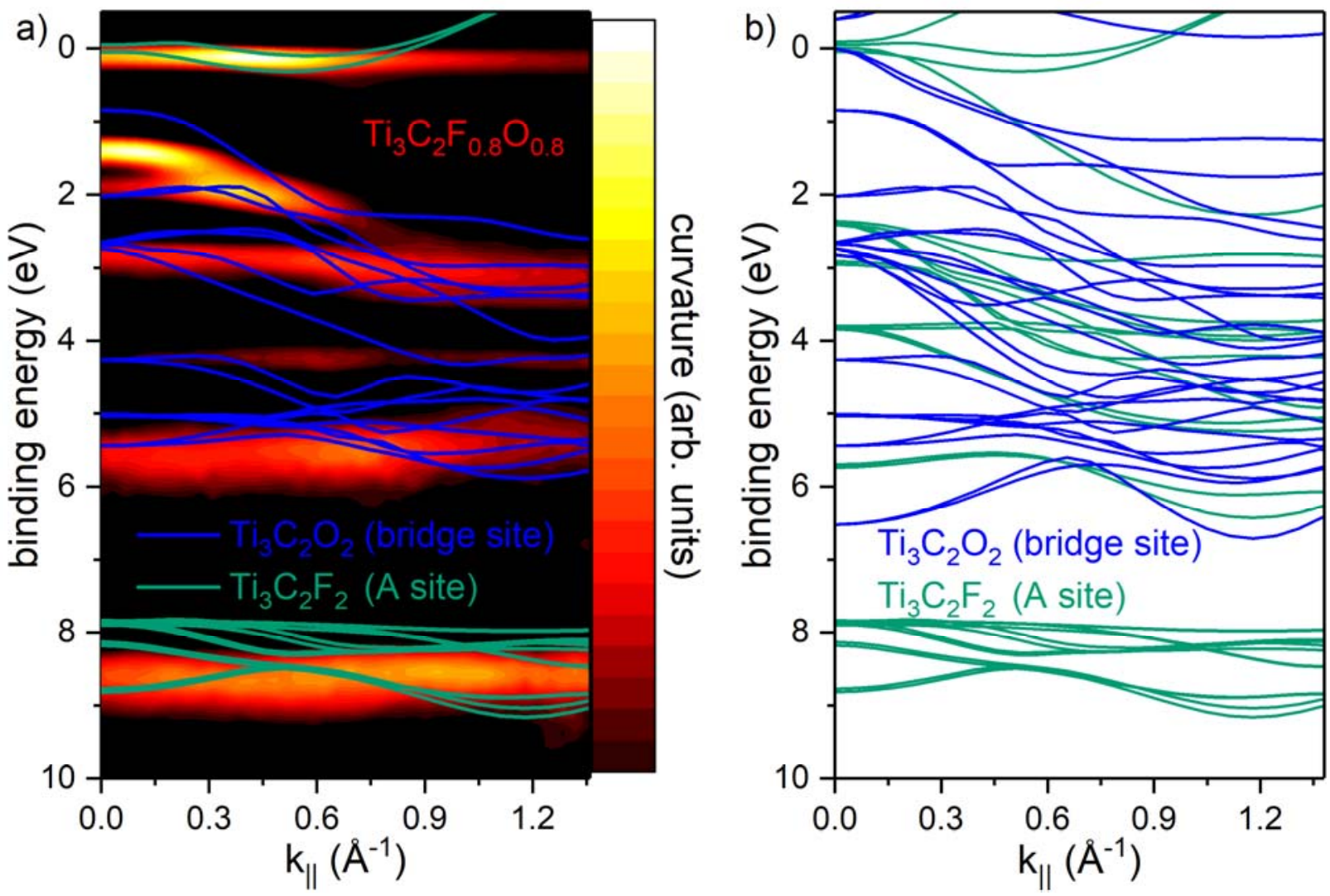

Figure 4: a) Measured ARPES curvature spectrum of $\mathrm{Ti}_{3} \mathrm{C}_{2} \mathrm{~F}_{0.8} \mathrm{O}_{0.8}$ (yellow-red), including a selected part of the calculated band structures of the high-symmetry $\Gamma-\mathrm{K}$ and $\Gamma-\mathrm{M}$ directions for $\mathrm{Ti}_{3} \mathrm{C}_{2} \mathrm{O}_{2}$ with $\mathrm{O}$ adsorbed only on the bridge site (blue) and for $\mathrm{Ti}_{3} \mathrm{C}_{2} \mathrm{~F}_{2}$ with $\mathrm{F}$ adsorbed only on the A-site (green). b) Full calculated band structures of the high-symmetry $\Gamma-\mathrm{K}$ and $\Gamma-\mathrm{M}$ directions for $\mathrm{Ti}_{3} \mathrm{C}_{2} \mathrm{O}_{2}$ with $\mathrm{O}$ adsorbed only on the bridge site (blue) and for $\mathrm{Ti}_{3} \mathrm{C}_{2} \mathrm{~F}_{2}$ with $\mathrm{F}$ adsorbed only on the A-site (greenish). The DFT calculations were done using the range-separated hybrid HSE06 functional and predict more bands than are observed in the curvature plot of the experimental data. 


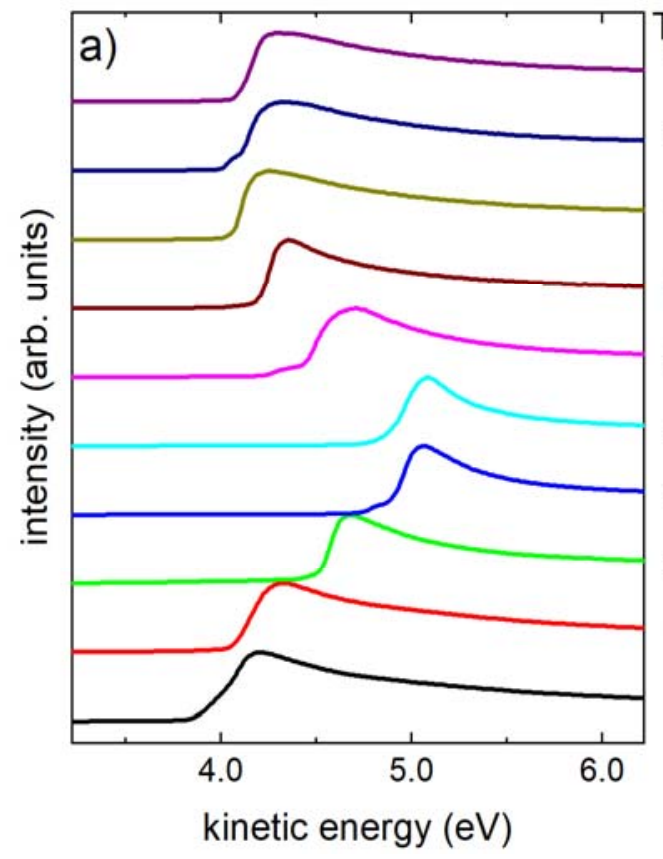

$7\left({ }^{\circ} \mathrm{C}\right)$
780
760
700
630
560
470
380
250
110
23

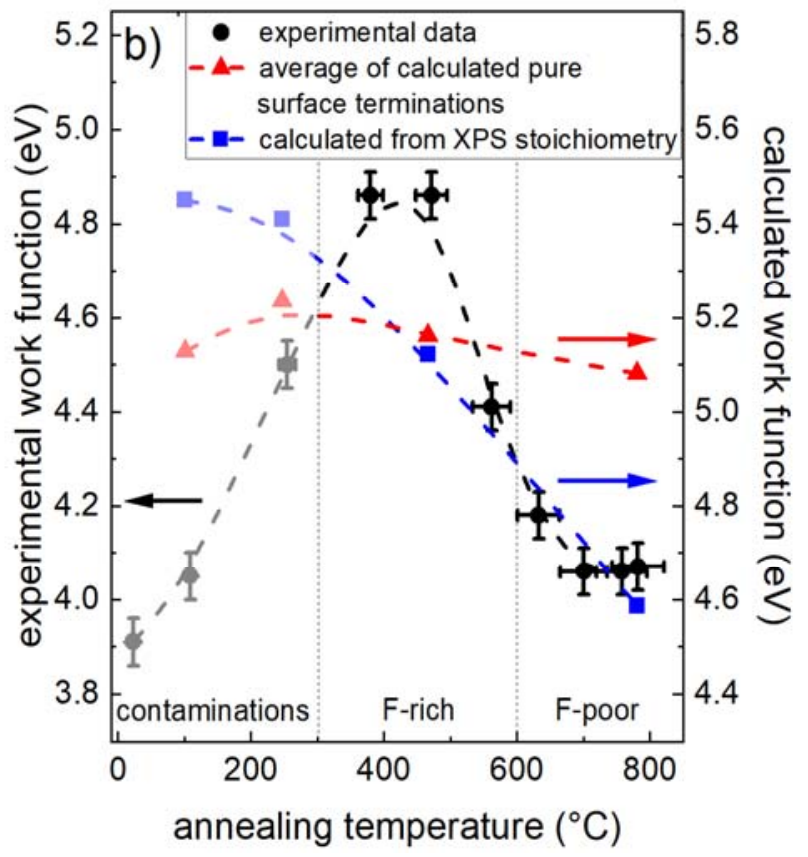

Figure 5: a) Secondary electron cut-off (SECO) spectra of $\mathrm{Ti}_{3} \mathrm{C}_{2} \mathrm{~T}_{\mathrm{x}}$ for different annealing temperatures. b) Work function values determined from the SECOs in a) as a function of annealing temperature. Work functions obtained from DFT for different surface terminations, calculated by using the real surface stoichiometry obtained from XPS (blue), and obtained by averaging the work functions of purely terminated $\mathrm{Ti}_{3} \mathrm{C}_{2} \mathrm{O}_{2}, \mathrm{Ti}_{3} \mathrm{C}_{2} \mathrm{~F}_{2}, \mathrm{Ti}_{3} \mathrm{C}_{2} \mathrm{OH}_{2}$ and $\mathrm{Ti}_{3} \mathrm{C}_{2}$ surfaces, weighted by the experimentally determined stoichiometry (red).

$3.9 \mathrm{eV}$, which increases to $4.8 \mathrm{eV}$ after annealing at $380^{\circ} \mathrm{C}$. One possible explanation could be a desorption of residual contaminations, like $\mathrm{LiF}$ or $\mathrm{Al}$ from the sample preparation process, or atmospheric water, carbon or carbon oxides, which are known to decrease the work function of clean metals via the push-back effect ${ }^{39,40}$. Another reason could be the desorption of $\mathrm{OH}$ surface terminations, which are predicted to significantly decrease the work function of $\mathrm{Ti}_{3} \mathrm{C}_{2}{ }^{14,41}$. Due to the low amount of $\mathrm{OH}$ groups as suggested by XPS results, the former is more likely. The maximum work function of $4.8 \mathrm{eV}$ is significantly lower than the predicted work function of $\mathrm{Ti}_{3} \mathrm{C}_{2} \mathrm{O}_{2}(\approx 6 \mathrm{eV})$, but significantly higher than the predicted work function of bare $\mathrm{Ti}_{3} \mathrm{C}_{2}(3.9 \mathrm{eV}) .{ }^{41}$ With desorption of fluorine at higher temperatures, the work function decreases again and saturates at ca. $4.1 \mathrm{eV}$. Work function values obtained from DFT calculations are also shown in Figure 5b). The red dots show an averaged work function, obtained by weighting the work function values of the homogeneously terminated surfaces $\left(\mathrm{Ti}_{3} \mathrm{C}_{2} \mathrm{O}_{2}, \mathrm{Ti}_{3} \mathrm{C}_{2} \mathrm{~F}_{2}, \mathrm{Ti}_{3} \mathrm{C}_{2} \mathrm{OH}_{2}\right.$ and $\left.\mathrm{Ti}_{3} \mathrm{C}_{2}\right)$ with the fraction of the different terminations obtained by evaluating the XPS core level peaks for the different annealing temperatures (Figure S8 in the SI). As can be seen, simply averaging the work function of the purely terminated surfaces does not sufficiently resemble the measured work function. A more involved procedure to calculate the work function was employed by constructing minimal supercells that accommodate the approximate surface stoichiometry measured by XPS and randomly distributing these terminations (see Table S3 in the SI for used surface terminations and supercell dimensions). This method more reliably accounts for the actual change in interlayer 
separation and local dipole distributions, resulting in a more accurate calculation of the overall surface dipole and work function. The results are shown in blue in Figure 5b). This approach leads to a significant reduction of the work function with reduced fluorine termination, in qualitative agreement with observations from experiment at higher annealing temperatures. Deviations between experiment and theory, especially at low annealing temperatures, stem most likely from contaminations, which cannot be included in the calculations. Additionally, we only considered the randomly distributed surface terminations in our work function calculations, neglecting the possibility of reorganization of surface terminations after thermal annealing. This leaves a more detailed investigation on the behavior of the surface terminations upon annealing as a future study.

\section{Conclusions}

Through comparison of XPS, angle-resolved UPS, and IPES measurements of $\mathrm{Ti}_{3} \mathrm{C}_{2} \mathrm{~T}_{\mathrm{x}}$ after different annealing temperatures with DFT calculations, we identified the adsorption sites of the surface terminations. Fluorine occupies the face-centered cubic adsorption site and oxygen initially occupies the bridge site and the hexagonal close-packed site, followed by a rearrangement to the face-centered cubic site after fluorine desorption at high annealing temperatures. Good agreement was found between calculated band structures for these adsorption sites and ARPES measurements, showing dispersion of more than $1 \mathrm{eV}$. Upon heating in vacuum, the sample work function increases from $3.9 \mathrm{eV}$ to $4.8 \mathrm{eV}$, most probably due to desorption of water, carbon-dominated contaminations, and $\mathrm{OH}$ species, and decreases again at higher temperatures to $4.1 \mathrm{eV}$ due to fluorine desorption. We find that the measured work function cannot be obtained by simply averaging the work function values of purely terminated $\mathrm{Ti}_{3} \mathrm{C}_{2} \mathrm{~T}_{\mathrm{x}}$ surfaces, but it can be better reproduced by modeling the real surface mixed-termination to account for local dipole effects. Our findings represent a solid foundation for the possibilities of determining the composition and electronic properties of MXenes with photoemission spectroscopy and will help to further explore and understand this new class of 2D materials.

\section{Acknowledgements}

The work in Berlin was supported by the Deutsche Forschungsgemeinschaft (DFG) Projektnummer 182087777 - SFB 951. N.C.F. was supported by the Department of Defense (DoD) through the National Defense Science \& Engineering Graduate Fellowship (NDSEG) Program. K.H., S.J.M., and Y.G. acknowledge support for research on synthesis and electronic properties of MXenes from the U.S. Department of Energy (DOE), Office of Science, Office of Basic Energy Sciences, grant \#DE-SC0018618.

\section{Supporting Information}

The supporting information include the following information: calculated binding energies for different oxygen adsorption sites; calculated lattice parameters and formation energies for different 
surface terminations on different adsorption sites; surface terminations and supercell dimensions used for work function calculations; core level and valence spectra for different annealing temperatures; temperature programmed desorption measurement; XPS survey spectra; UPS background subtraction procedure; Brillouin zone schematic and explanations for observation of band dispersion mainly along the high-symmetry directions; calculated band structures for different surface terminations and adsorption sites; IPES spectra; summary surface terminations from XPS; AFM image; dynamic light scattering measurement.

\section{References}

(1) Novoselov, K.S., Jiang, D., Schedin, F., Booth, T.J., Khotkevich, V.V., Morozov, S.V. and Geim, A.K. Two-dimensional atomic crystals. Proceedings of the National Academy of Sciences, 2005, 102, 10451-10453.

(2) Nicolosi, V., Chhowalla, M., Kanatzidis, M.G., Strano, M.S. and Coleman, J.N. Liquid Exfoliation of Layered Materials. Science, 2013, 340, 1226419.

(3) Naguib, M., Kurtoglu, M., Presser, V., Lu, J., Niu, J., Heon, M., Hultman, L., Gogotsi, Y. and Barsoum, M.W. Two-Dimensional Nanocrystals Produced by Exfoliation of $\mathrm{Ti}_{3} \mathrm{AlC}_{2}$. Advanced Materials, 2011, 23, 4248-4253.

(4) Barsoum, M.W. MAX Phases: Properties of Machinable Ternary Carbides and Nitrides. Wiley VCH Verlag GmbH \& Co. KGaA, Weinheim, Germany, 2013.

(5) Naguib, M., Mashtalir, O., Carle, J., Presser, V., Lu, J., Hultman, L., Gogotsi, Y. and Barsoum, M.W. Two-Dimensional Transition Metal Carbides. ACS Nano, 2012, 6, 1322 1331.

(6) Hantanasirisakul, K. and Gogotsi, Y. Electronic and Optical Properties of Two-Dimensional Transition Metal Carbides and Nitrides (MXenes). Advanced Materials, 2018, 30, 1804779.

(7) Anasori, B., Lukatskaya, M.R. and Gogotsi, Y. 2D metal carbides and nitrides (MXenes) for energy storage. Nature Reviews Materials, 2017, 2, 16098.

(8) Shahzad, F., Alhabeb, M., Hatter, C.B., Anasori, B., Man Hong, S., Koo, C.M. and Gogotsi, Y. Electromagnetic interference shielding with 2D transition metal carbides (MXenes). Science, 2016, 353, 1137-1140.

(9) Kim, S.J., Koh, H.-J., Ren, C.E., Kwon, O., Maleski, K., Cho, S.-Y., Anasori, B., Kim, C.-K., Choi, Y.-K., Kim, J., et al. Metallic $\mathrm{Ti}_{3} \mathrm{C}_{2} \mathrm{~T}_{\mathrm{x}}$ MXene Gas Sensors with Ultrahigh Signalto-Noise Ratio. ACS Nano, 2018, 12, 986-993.

(10) Ma, Y., Liu, N., Li, L., Hu, X., Zou, Z., Wang, J., Luo, S. and Gao, Y. A highly flexible and sensitive piezoresistive sensor based on MXene with greatly changed interlayer distances. Nature Communications, 2017, 8, 1207. 
(11) Huang, K., Li, Z., Lin, J., Han, G. and Huang, P. Two-dimensional transition metal carbides and nitrides (MXenes) for biomedical applications. Chem. Soc. Rev., 2018, 47, 51095124.

(12) Römer, F.M., Wiedwald, U., Strusch, T., Halim, J., Mayerberger, E., Barsoum, M.W. and Farle, M. Controlling the conductivity of $\mathrm{Ti}_{3} \mathrm{C}_{2}$ MXenes by inductively coupled oxygen and hydrogen plasma treatment and humidity. RSC Adv., 2017, 7, 13097-13103.

(13) Hart, J.L., Hantanasirisakul, K., Lang, A.C., Anasori, B., Pinto, D., Pivak, Y., Omme, J.T. van, May, S.J., Gogotsi, Y. and Taheri, M.L. Control of MXenes' Electronic Properties Through Termination and Intercalation. Nature Communications, 2019, 10, 522.

(14) Khazaei, M., Arai, M., Sasaki, T., Ranjbar, A., Liang, Y. and Yunoki, S. OH-terminated two-dimensional transition metal carbides and nitrides as ultralow work function materials. Phys. Rev. B, 2015, 92, 075411.

(15) Khazaei, M., Arai, M., Sasaki, T., Chung, C.-Y., Venkataramanan, N.S., Estili, M., Sakka, Y. and Kawazoe, Y. Novel Electronic and Magnetic Properties of Two-Dimensional Transition Metal Carbides and Nitrides. Advanced Functional Materials, 2013, 23, 2185 2192.

(16) Khazaei, M., Ranjbar, A., Arai, M., Sasaki, T. and Yunoki, S. Electronic properties and applications of MXenes: a theoretical review. J. Mater. Chem. C, 2017, 5, 2488-2503.

(17) Magnuson, M., Halim, J. and Näslund, L.-Å. Chemical bonding in carbide MXene nanosheets. Journal of Electron Spectroscopy and Related Phenomena, 2018, 224, 27-32.

(18) Wang, Z., Kim, H. and Alshareef, H.N. Oxide Thin-Film Electronics using All-MXene Electrical Contacts. Advanced Materials, 2018, 30, 1706656.

(19) Alhabeb, M., Maleski, K., Anasori, B., Lelyukh, P., Clark, L., Sin, S. and Gogotsi, Y. Guidelines for Synthesis and Processing of Two-Dimensional Titanium Carbide $\left(\mathrm{Ti}_{3} \mathrm{C}_{2} \mathrm{~T}_{\mathrm{x}}\right.$ MXene). Chemistry of Materials, 2017, 29, 7633-7644.

(20) Kresse, G. and Furthmüller, J. Efficient iterative schemes for ab initio total-energy calculations using a plane-wave basis set. Phys. Rev. B, 1996, 54, 11169-11186.

(21) Perdew, J.P., Burke, K. and Ernzerhof, M. Generalized Gradient Approximation Made Simple. Phys. Rev. Lett., 1996, 77, 3865-3868.

(22) Kresse, G. and Joubert, D. From ultrasoft pseudopotentials to the projector augmented-wave method. Phys. Rev. B, 1999, 59, 1758-1775.

(23) Xie, Y., Naguib, M., Mochalin, V.N., Barsoum, M.W., Gogotsi, Y., Yu, X., Nam, K.-W., Yang, X.-Q., Kolesnikov, A.I. and Kent, P.R.C. Role of Surface Structure on Li-Ion Energy Storage Capacity of Two-Dimensional Transition-Metal Carbides. Journal of the American Chemical Society, 2014, 136, 6385-6394. 
(24) Wu, F., Luo, K., Huang, C., Wu, W., Meng, P., Liu, Y. and Kan, E. Theoretical understanding of magnetic and electronic structures of $\mathrm{Ti}_{3} \mathrm{C}_{2}$ monolayer and its derivatives. Solid State Communications, 2015, 222, 9-13.

(25) Heyd, J., Scuseria, G.E. and Ernzerhof, M. Hybrid functionals based on a screened Coulomb potential. The Journal of Chemical Physics, 2003, 118, 8207-8215.

(26) Krukau, A.V., Vydrov, O.A., Izmaylov, A.F. and Scuseria, G.E. Influence of the exchange screening parameter on the performance of screened hybrid functionals. The Journal of Chemical Physics, 2006, 125, 224106.

(27) Sun, J., Ruzsinszky, A. and Perdew, J.P. Strongly Constrained and Appropriately Normed Semilocal Density Functional. Phys. Rev. Lett., 2015, 115, 036402.

(28) Zhang, Y., Sun, J., Perdew, J.P. and Wu, X. Comparative first-principles studies of prototypical ferroelectric materials by LDA, GGA, and SCAN meta-GGA. Phys. Rev. B, 2017, 96, 035143.

(29) Persson, I., Näslund, L.-Å., Halim, J., Barsoum, M.W., Darakchieva, V., Palisaitis, J., Rosen, J. and Persson, P.O.A. On the organization and thermal behavior of functional groups on $\mathrm{Ti}_{3} \mathrm{C}_{2}$ MXene surfaces in vacuum. 2D Materials, 2018, 5, 015002.

(30) Kong, F., He, X., Liu, Q., Qi, X., Zheng, Y., Wang, R. and Bai, Y. Improving the electrochemical properties of $\mathrm{MXene} \mathrm{Ti}_{3} \mathrm{C}_{2}$ multilayer for $\mathrm{Li}$-ion batteries by vacuum calcination. Electrochimica Acta, 2018, 265, 140-150.

(31) Diebold, U. and Madey, T.E. TiO 2 by XPS. Surface Science Spectra, 1996, 4, 227-231.

(32) Kurtz, R.L. and Henrich, V.E. Comparison of Ti 2p Core-Level Peaks from $\mathrm{TiO}_{2}, \mathrm{Ti}_{2} \mathrm{O}_{3}$, and Ti Metal, by XPS. Surface Science Spectra, 1998, 5, 179-181.

(33) Shen, C., Wang, L., Zhou, A., Wang, B., Wang, X., Lian, W., Hu, Q., Qin, G. and Liu, X. Synthesis and Electrochemical Properties of Two-Dimensional RGO/Ti ${ }_{3} \mathrm{C}_{2} \mathrm{~T}_{\mathrm{x}}$ Nanocomposites. Nanomaterials, 2018, 8.

(34) Shah, S.A., Habib, T., Gao, H., Gao, P., Sun, W., Green, M.J. and Radovic, M. Templatefree 3D titanium carbide $\left(\mathrm{Ti}_{3} \mathrm{C}_{2} \mathrm{~T}_{\mathrm{x}}\right)$ MXene particles crumpled by capillary forces. Chem. Commun., 2017, 53, 400-403.

(35) Cao, Y., Deng, Q., Liu, Z., Shen, D., Wang, T., Huang, Q., Du, S., Jiang, N., Lin, C.-T. and $\mathrm{Yu}$, J. Enhanced thermal properties of poly(vinylidene fluoride) composites with ultrathin nanosheets of MXene. RSC Adv., 2017, 7, 20494-20501.

(36) Park, S., Mutz, N., Schultz, T., Blumstengel, S., Han, A., Aljarb, A., Li, L.-J., ListKratochvil, E.J.W., Amsalem, P. and Koch, N. Direct determination of monolayer $\mathrm{MoS}_{2}$ and $\mathrm{WSe}_{2}$ exciton binding energies on insulating and metallic substrates. 2D Materials, 2018, 5, 025003. 
(37) Zhou, S.Y., Gweon, G.-H., Spataru, C.D., Graf, J., Lee, D.-H., Louie, S.G. and Lanzara, A. Coexistence of sharp quasiparticle dispersions and disorder features in graphite. Phys. Rev. B, 2005, 71, 161403.

(38) Zhang, P., Richard, P., Qian, T., Xu, Y.-M., Dai, X. and Ding, H. A precise method for visualizing dispersive features in image plots. Review of Scientific Instruments, 2011, 82, 043712.

(39) Heras, J.M. and Viscido, L. Work function changes upon water contamination of metal surfaces. Applications of Surface Science, 1980, 4, 238-241.

(40) Helander, M.G., Greiner, M.T., Wang, Z.B. and Lu, Z.H. Pitfalls in measuring work function using photoelectron spectroscopy. Applied Surface Science, 2010, 256, $2602-$ 2605.

(41) Caffrey, N.M. Effect of mixed surface terminations on the structural and electrochemical properties of two-dimensional $\mathrm{Ti}_{3} \mathrm{C}_{2} \mathrm{~T}_{2}$ and $\mathrm{V}_{2} \mathrm{CT}_{2}$ MXenes multilayers. Nanoscale, 2018, $10,13520-13530$.
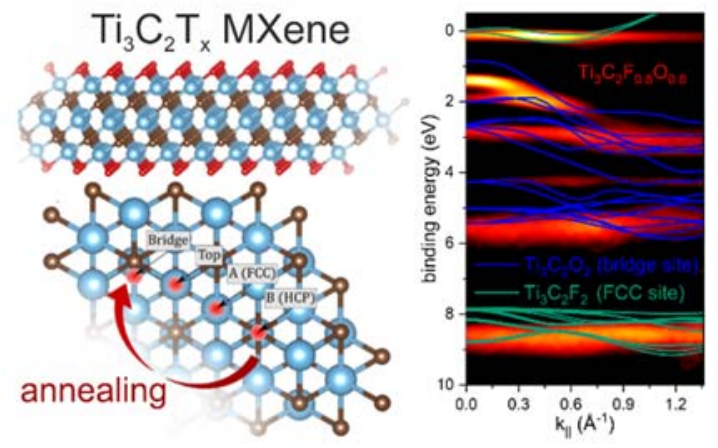

TOC Figure 